\title{
R Peak Detection using Wavelet
}

\author{
Amana Yadav \\ ECE Deptt. FET, Manav Rachna International \\ University \\ Sec.43, Arravali Hills, Faridabad, 121001
}

\author{
Naresh Grover, PhD \\ ECE Deptt. FET, Manav Rachna International \\ University \\ Sec.43, Arravali Hills, Faridabad, 121001
}

\begin{abstract}
ECG is very crucial and important tool to detect the cardiac problems. It has all the information related to the electrical activities of heart. This also has the information of normal and abnormal activities for the detection of the diseases. So it is essential and important to detect the accurate R-peaks in QRS complex, especially when the results are to be used for clinical applications. Hence in a long-term ECG signal, automatic R-peaks detection is very essential to diagnose cardiac disorders. In this paper we proposed a robust technique to detect R-peak which uses Wavelet Transform. The proposed R Peak detector is consists of a wavelet filter banks, a noise detector with zero-crossing points, multi-scaled product algorithm and soft-threshold algorithm.
\end{abstract}

\section{Keywords}

ECG, R-peak detection, QRS complex, P-QRS-T waves, Filters, MATLAB.

\section{INTRODUCTION}

Electrocardiogram (ECG) is the recording and register of electrical activity of the heart [1]. The ECG has the frequency from (0.1-150) Hz. It is very useful to detect cardiac disorders [2]. For that ECG feature extraction is important step. In ECG feature extraction, the first step is R peak detection. It contains highest amplitude and necessary information. Therefore exact $\mathrm{R}$ peak detection is very essential in ECG signal analysis [3].

A typical ECG consists of different segments of ECG as P wave, a T wave, a QRS complex and a $U$ wave. [4]. ECG is shown in fig1.

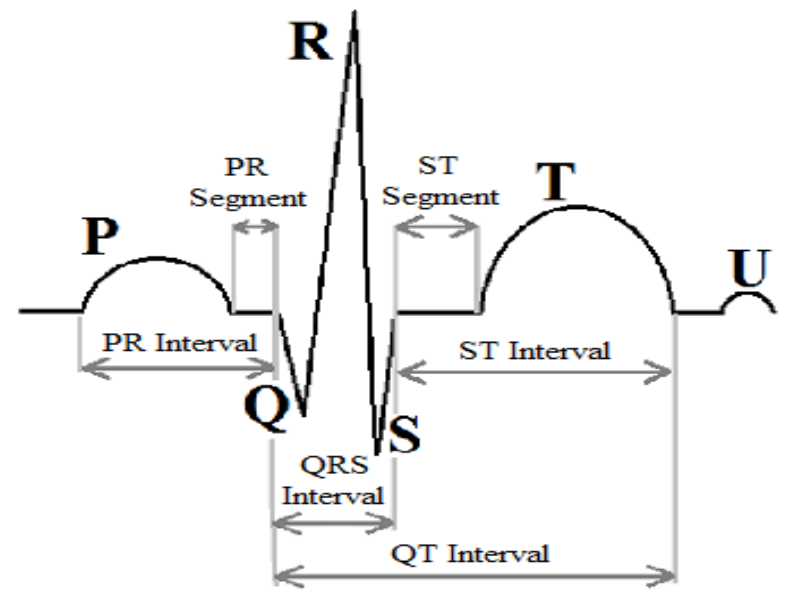

Fig.1 A normal ECG signal, consists of a $P$ wave, a $Q R S$ complex and a $T$ wave [5]

Our proposed work deals with ECG analysis and R peak detection. Many methods have been designed to detect the $\mathrm{R}$ peaks. Here we are proposing the method using wavelet transform. Wavelet transform gives the information of both time and frequency. Hence the wavelet has been used for the extraction of relevant information [6]. The ECG signal is decomposed into a set of frequency band using wavelet transform [7]. Our proposed work shows the sharp R peaks. To implement this algorithm MATLAB 8.2 version has been used.

This method for $\mathrm{R}$ peak detection is validated using the firstchannel of the 48 ECG records of the MIT-BITH arrhythmia database [8]. Using this method average detection accuracy of $99.997 \%$, positive predictivity of $99.99 \%$ and sensitivity of $99.75 \%$ have been achieved.

The paper is organized as follows: Section 2, describes the five-stage R-peak detection methodology in detail. This section provides the detail of our proposed peak-finding technique. Section 3, represents the experimental results to show the quality of the technique. Finally, in Section 4, we conclude our work and also present future scope.

\section{PROPOSED METHODOLOGY}

Our proposed $\mathrm{R}$ peak detection method is shown in Fig. 2 which consists of four stages, as Wavelet filter bank, Noise detector having zero crossing point and a $\mathrm{R}$ peak detector stage consists of multi scaled product and soft threshold. Finally we get $\mathrm{R}$ peaks. Filter is used to decompose the signal into low frequency and high frequency. We obtained four signals WF1, WF2, WF3, and WF4 from wavelet decomposer then put a multi scaled product section where one multiplier and two multiplexers are used. One of the filter output is selected depending on the signal ND from noise detector. After that this signal is applied to soft threshold which removes the failure occurs in the peaks and finally we get $R$ peaks. The block diagram of our proposed methodology is shown in Fig 2.

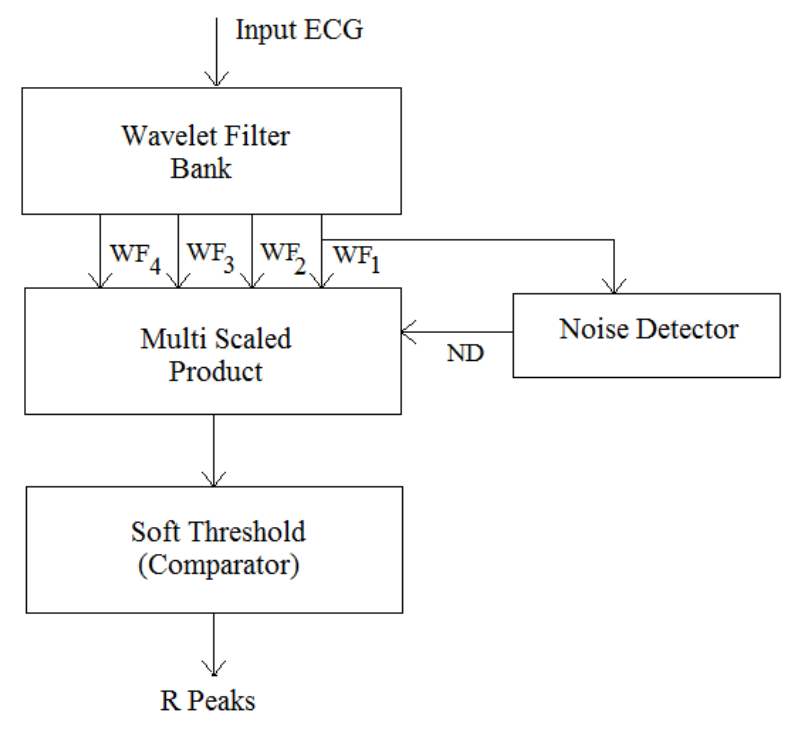

Fig. 2 R-peak Detection Technique 
The detailed discussions of every block are as follows:

MIT-BIH database are used for input ECG

\subsection{Wavelet}

Wavelet transform has been used to find the parameter of ECG. In wavelet analysis to study about the signal, the signal is simplify into its fraction [9]. There are two filters in Discrete Wavelet Transform (DWT) first is a low pass filter (LPF) and second a high pass filter (HPF). Both the filters are utilized to divide the signal into many portions [10]. There are two coefficients Detailed and Approximation. Approximation coefficients are output coefficient of LPF and detailed coefficients are HPF. For second-level decomposition these approximation signal is passed to another level LPF and HPF. In this way the signal is divided into its different components at different levels of scale [11]. In the study of wavelet the signal is divided into the frequency bands. In the synthesis of wavelet the divided signal is reproduced again into the actual bands using filter [12].

The convolution of the wavelet function $\psi(\mathrm{t})$ with the signal $\mathrm{x}(\mathrm{t})$ is wavelet transform. The discrete wavelets which are orthonormal dyadic are associated with scaling functions $\varphi(t)$. The signal can be convolved with the scaling function to produce approximation coefficients [13]. The discrete wavelet transform (DWT) can be written as Tm,n

$$
T_{m, n}=\int_{-\infty}^{\infty} x(t) \varphi_{m, n}(t) d(t)
$$

By selecting an orthonormal wavelet basis $\psi_{\mathrm{m}, \mathrm{n}}(\mathrm{t})$ we can again reconstruct the original signal. The signal's Approximation coefficient at the $\mathrm{m}$ scale and $\mathrm{n}$ location can be represented as

$$
S_{m, n} \int_{-\infty}^{\infty} x(\mathrm{t}) \varphi_{\mathrm{m}, \mathrm{n}}(\mathrm{t}) d(\mathrm{t})
$$

In practice the discrete input signal having $\mathrm{N}$ finite length. Hence the range of scales which can be tested is $0<\mathrm{m}$. A discrete signal approximation can be represented as

$$
x_{0}(t)=x_{M}(t)+\sum_{m=1}^{M} d_{m}(t)
$$

mean approximation of signal at scale $\mathrm{M}$ is

$$
x_{0}(t)=x_{M}(t)+\sum d_{m}(t)
$$

and for finite length signal, detail signal approximation which corresponds to scale $\mathrm{m}$ is

$$
\mathrm{d}_{\mathrm{m}}(\mathrm{t})=\sum_{\mathrm{n}=0}^{\mathrm{M}-\mathrm{m}} \mathrm{T}_{\mathrm{m}, \mathrm{n}} \varphi_{\mathrm{m}, \mathrm{n}}(\mathrm{t})
$$

The Signal approximation at a particular scale is the mixture of the detail and approximation at the next lower scale.

$$
x_{m}(t)=x_{m-1}(t)-d_{m}(t)
$$

We got four signals WF1, WF2, WF3, and WF4 from wavelet decomposer.

\subsection{Multi Scaled Product}

In Multi scaled product block one multiplier and two multiplexers are used where output of filter bank goes as input to this block and depending on the signal ND from noise detector one of the output has been selected. Multi scaled product of the output of wavelet filter bank is expressed as

$$
\mathrm{MP}_{\mathrm{I}}=\Pi_{\mathrm{I}}\left|\mathrm{WF}_{\mathrm{I}}\right|
$$

\subsection{Soft Threshold:}

In order to remove the failure occurred in the QRS complex detection, the soft-threshold algorithm, which uses variable thresholds rather than a hard threshold, has been used.

\section{RESULTS AND DISCUSSION}

We used the MIT-BIH arrhythmia database to evaluate the proposed R-peak detection technique. MIT-BIH database contains 48 half an hour of two channel ECG recordings with sampling frequency $360 \mathrm{~Hz}$. The proposed algorithm was using MATLAB version 8.2 and tested on numerous ECG signals taken from the database of MIT-BIH arrhythmia [14]. True-positive (TP) for the case when a R-peak is correctly achieved is calculated by this algorithm.

We use following parameter to measure the performance of our algorithm.

Detection accuracy which is

$$
\text { Accuracy }(\text { Acc })=T_{\mathrm{P}} /\left(\mathrm{T}_{\mathrm{P}}+\mathrm{F}_{\mathrm{P}}+\mathrm{F}_{\mathrm{N}}\right) \times 100 \%
$$

Sensitivity is

$$
\text { Sensitivity }=T_{P} /\left(T_{P}+F_{N}\right) \times 100 \%
$$

Positive Predictivity is

$$
\text { Positive Predictivity }=T_{P} /\left(T_{P}+F_{P}\right) \times 100 \%
$$

This method gave an improved R-peak detection under various noises.

The waveforms of few stages of the proposed method using five samples of the MIT/BIH database are shown in Figure 3, 4, 5, 6 and 7, where (a) displays the original Input ECG signal. The waveform shown in (b) represents the detected Rpeaks using the given method.
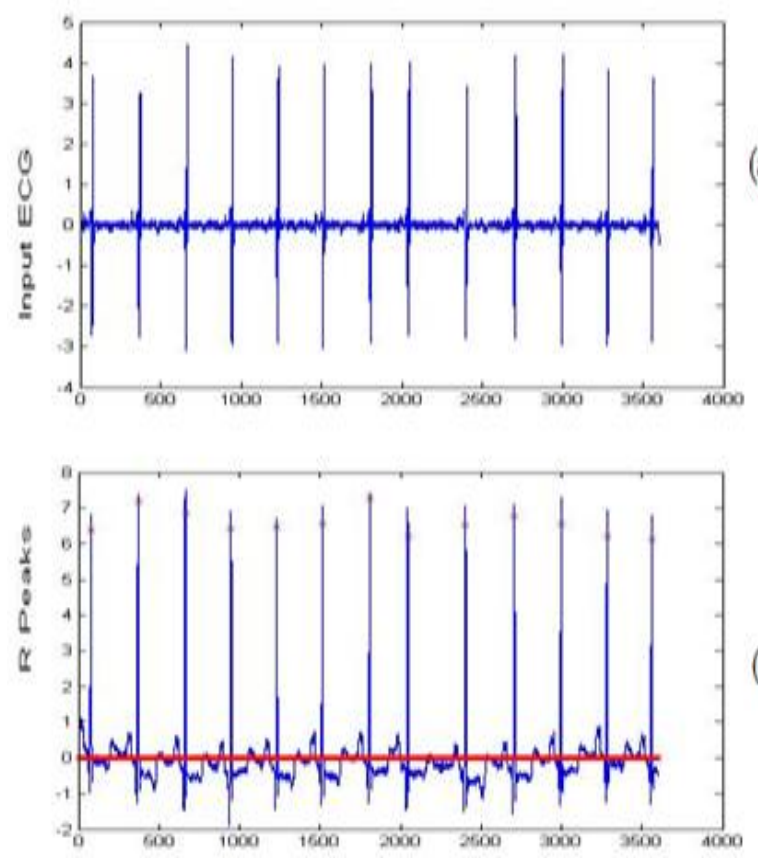

Number of Samples

Fig. 3 Proposed R peaks detector for record 100: (a) the actual input ECG signal and (b) output of obtained Rpeaks.

where I is the sub-set of WFB outputs. 

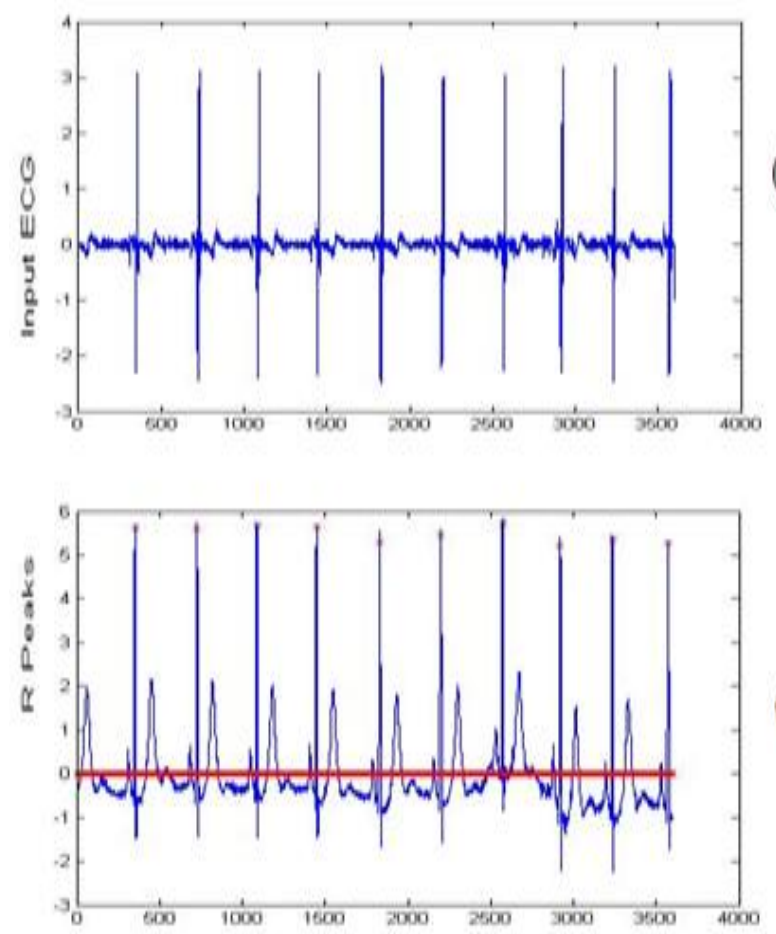

Number of Samples

Fig. 4 Proposed $R$ peaks detector for record 106: (a) the actual input ECG signal and (b) output of obtained Rpeaks.
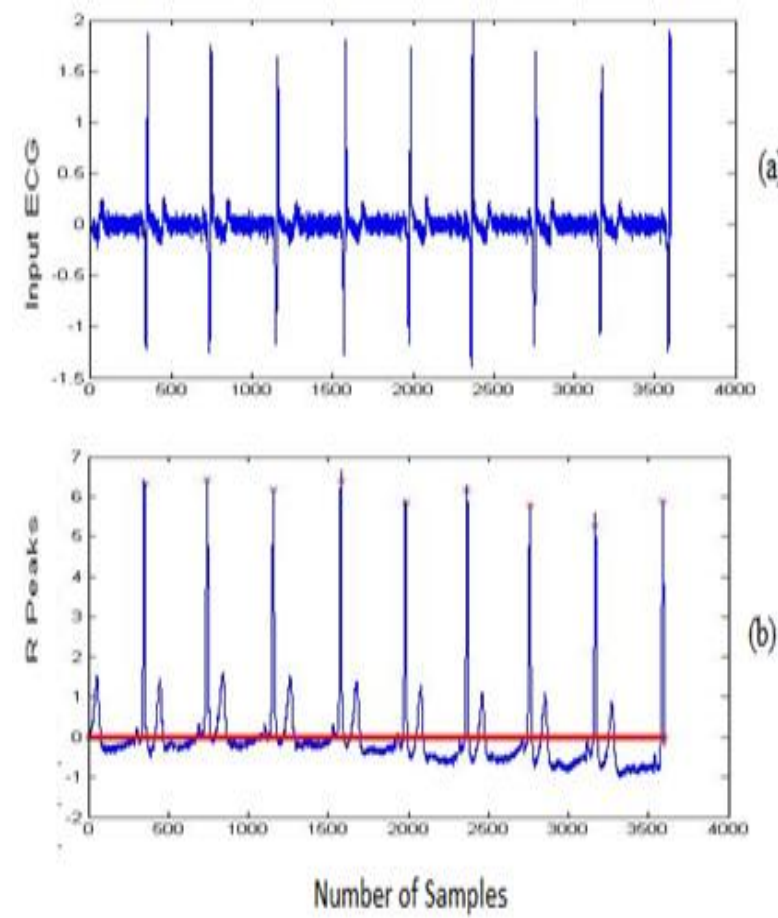

Fig. 5 Proposed R peaks detector for record 202: (a) the actual input ECG signal and (b) output of obtained Rpeaks. (a)

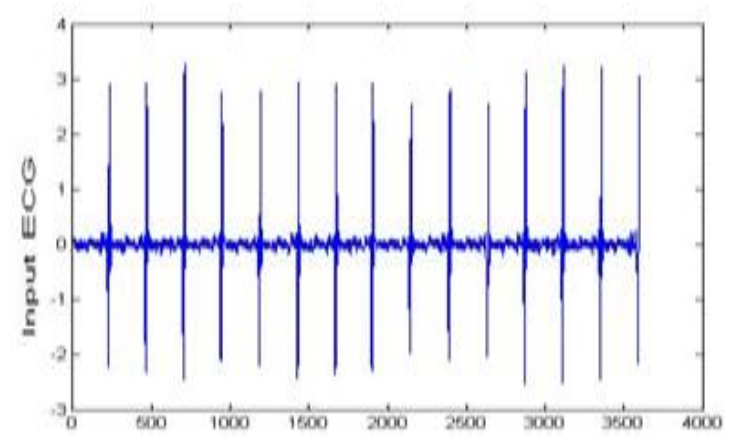

(a)

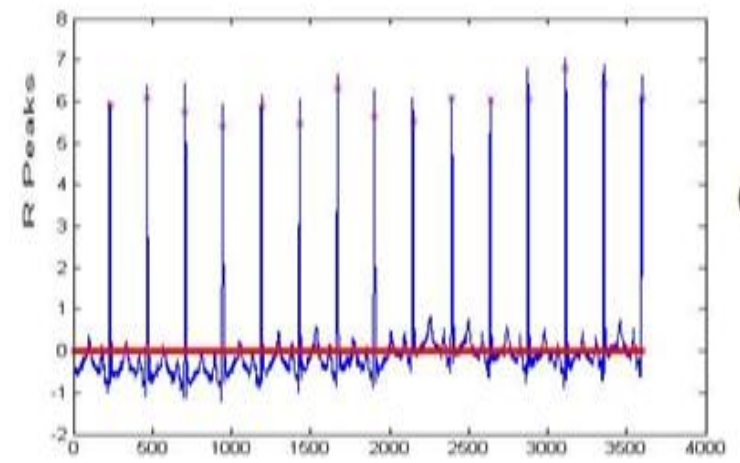

Number of Samples

Fig. 6 Proposed R peaks detector for record 205: (a) the actual input ECG signal and (b) output of obtained Rpeaks.

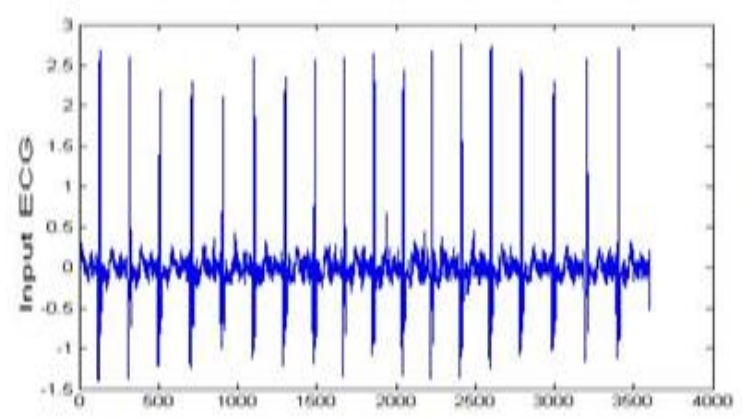

(a)

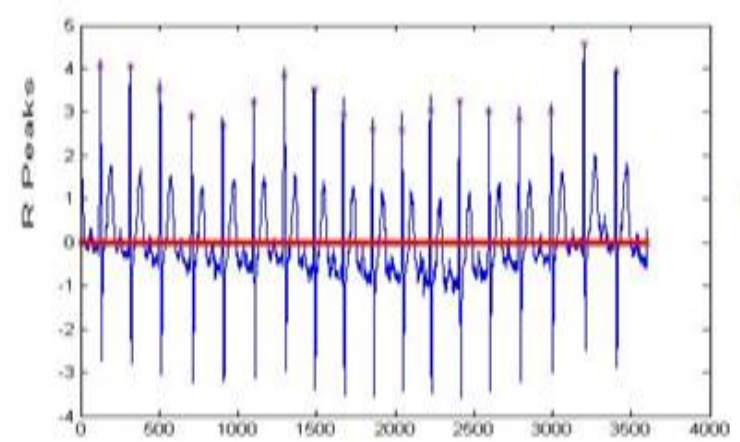

Number of Samples

Fig. 7 Proposed R peaks detector for record 215: (a) the actual input ECG signal and (b) output of obtained Rpeaks.

(b)

(b) 
From the table it can be concluded that an average accuracy of $99.9972 \%$, a positive predictivity of $99.99 \%$ and a sensitivity of $99.7538 \%$ are achieved from our technique.

Performance parameter have also been measured for five records of MIT-BIH database and shown them in table 1.

Table No.1 Performance assessment of given R-peak detection method using MIT-BIH arrhythmia database

\begin{tabular}{|c|c|c|c|c|c|c|c|}
\hline $\begin{array}{l}\text { ECG } \\
\text { Rec- } \\
\text { ord } \\
\text { No. }\end{array}$ & $\begin{array}{l}\text { Total } \\
\text { Beats }\end{array}$ & $\begin{array}{l}\text { False } \\
\text { Posi- } \\
\text { tive } \\
\text { Beats }\end{array}$ & $\begin{array}{l}\text { False } \\
\text { Nega- } \\
\text { tive } \\
\text { Beats }\end{array}$ & $\begin{array}{l}\text { DER } \\
(\%)\end{array}$ & $\begin{array}{l}\text { Sensi- } \\
\text { tivity } \\
(\%)\end{array}$ & $\begin{array}{l}\text { (+Ve) } \\
\text { Predi- } \\
\text { tivity } \\
(\%)\end{array}$ & $\begin{array}{l}\text { Accu- } \\
\text { racy } \\
(\%)\end{array}$ \\
\hline 100 & 2273 & 0 & 0 & 0 & 100 & 100 & 100 \\
\hline 102 & 2187 & 0 & 5 & $\begin{array}{c}0.22 \\
82\end{array}$ & 99.77 & 100 & 99.99 \\
\hline 103 & 2084 & 0 & 9 & $\begin{array}{c}0.43 \\
06\end{array}$ & $\begin{array}{c}99.56 \\
9\end{array}$ & 100 & $\begin{array}{c}99.99 \\
8\end{array}$ \\
\hline 111 & 2124 & 1 & 12 & $\begin{array}{c}0.60 \\
98\end{array}$ & 99.43 & 99.95 & $\begin{array}{c}99.99 \\
8\end{array}$ \\
\hline 113 & 1795 & 0 & 0 & 0 & 100 & 100 & 100 \\
\hline
\end{tabular}

\section{CONCLUSION}

The extraction of ECG parameter is very important to detect the cardiac abnormality i..e. Tachycardia and Bradycardia. As $\mathrm{R}$ peak contains maximum information so detection of accurate $\mathrm{R}$ peak is very important. Hence we have designed an automated $\mathrm{R}$ peak detection method from ECG signal which uses four stages methodology in this paper.

The results achieved from the proposed technique has been shown and discussed. The efficiency of the proposed technique have been tested on the standard arrhythmia database of MIT-BIH and final performance was calculated in terms of following parameters, i.e. the number of true positives, false positives and false negatives for every record. The given R-peak detector technique has $99.99 \%$ accuracy, $99.75 \%$ sensitivity and $99.99 \%(+\mathrm{Ve})$ predictivity. The results have proven that this method to find $\mathrm{R}$ Peak has better efficiency for pathological or noisy signals.

This technique can be updated in future for real time ECG applications.

\section{REFERENCES}

[1] P. Trivedi, S. Ayub, "Detection of $R$ Peak in Electrocardiogram", International Journal of Computer Applications (0975 - 8887) Volume 97 - No.20, July 2014, pp 10-14.

[2] M. S. Manikandan, K.P. Soman "A novel method for detecting R-peaks in electrocardiogram (ECG) signal",
Biomedical Signal Processing and Control 7 (2012) 118 128.

[3] C. Meyer, J.F. Gavela, M. Harris, Combining algorithms in automatic detection of QRS complexes in ECG signals, IEEE Trans. Inf. Technol. Biomed. 10 (3) (2006) 468-475.

[4] Pahlm O., Sörnmo L., "Software QRS detection in ambulatory monitoring - a review", Med. Biol. Eng. Comput. 22 (1984) 289-297.

[5] S. Thulasi Prasad, Dr. S. Varadarajan, "Heart Rate Detection using Hilbert Transform", International Journal of Research in Engineering and Technology, Volume 02, Issue 08, Aug-2013, pages 508-513,

[6] B. Abibullaev, H.D. Seo, A New QRS detection method using wavelets and artificial neural networks, J. Med. Syst. (2010), doi:10.1007/s10916-009-9405-3.

[7] I. Nouira, A. Ben Abdallah, Mohamed H. Bedoui, and Mohamed Dogui, "A Robust R Peak Detection Algorithm Using Wavelet Transform for Heart Rate Variability Studies", International Journal on Electrical Engineering and Informatics - Volume 5, Number 3, September 2013 pp 270-284.

[8] P.S. Hamilton, W.J. Tompkins, "Quantitative investigation of QRS detection rules using the MIT/BIH arrhythmia database", IEEE Trans. Biomed. Eng. 33 (1986) 1157-1165

[9] A. Ghaari a, H. Golbayani, "A new mathematical based QRS detector using continuous wavelet transform", Science Direct Computers and Electrical Engineering 34 pp. 81-91, May 2008.

[10] Gordan Cornelia, Reiz Romulus, "ECG signals processing using Wavelets", IEEE, proceedings of the fifth laserd International conference May 2005.

[11] P. Manimegalai, R. Dhanapal, Dr. K. Thanushkodi, Real Time Implementation of QRS Complex Extraction Using Discrete Wavelets, International Journal of Emerging Technology and Advanced Engineering, Volume 2, Issue 2, February 2012.

[12] Ramakrishna and S.Saha, "ECG coding by wavelet based linear prediction", IEEE Transactions on Biomedical Engineering, vol.44, no. 12, pp. 1253-1261, Dec, 1997.

[13] Chia-Hung Lin, Yi Chun Du, Tainsong Chen, "Adaptive wavelet network for multiple cardiac arrhythmias recognition", Science Direct, Expert Systems with Applications, pp 2601-2611, May 2008.

[14] MIT-BIH (http://www.physionet.org). 